\title{
Incidence of hiatus hernia and gastro-oesophageal reflux in 1030 prospective barium meal examinations in adult Nigerians
}

\author{
O. O. BASSEY, E. E. EYO, AND G. A. AKINHANMI \\ From the Departments of Surgery and Radiodiagnosis, College of Medicine, University of Lagos, \\ P.M. Bag 12003, Lagos, Nigeria
}

\begin{abstract}
Bassey, O. O., Eyo, E. E., and Akinhanmi, G. A. (1977). Thorax, 32, 356-359. Incidence of hiatus hernia and gastro-oesophageal reflux in 1030 prospective barium meal examinations in adult Nigerians. A prospective study to determine the incidence of hiatus hernia and gastrooesophageal reflux in 1030 consecutive symptomatic adult Nigerian patients undergoing barium meal examination is reported. The results show a very low incidence of hiatus hernia $(0 \cdot 39 \%)$ and an equally low incidence of gastro-oesophageal reflux $(2 \cdot 2 \%)$ when compared with similar studies in Europe and America. There was a high incidence of duodenal ulcer $(23 \cdot 3 \%)$ and a low incidence of gastric ulcer $(1 \cdot 8 \%)$, the duodenal/gastric ulcer ratio of $12 \cdot 1: 1$ being much higher than in Europe.
\end{abstract}

Hiatus hernia is a very common condition in the western world. Gastro-oesophageal reflux is commonly associated with sliding hiatus hernia and is a common cause of dyspepsia there (Barrett, 1952; Atkinson, 1967; Beeley and Warner, 1972; Burkitt and James, 1973). In these countries hiatus hernia may be found in up to $50 \%$ of symptomatic patients during barium meal examinations (Stein and Finkelstein, 1960). However, experience in Nigeria (Davey, 1968; Bassey, 1975) as well as in other parts of Africa (Trowell, 1960; Burkitt 1971; Burkitt and James, 1973) is that hiatus hernia and symptomatic gastro-oesophageal reflux are both uncommon.

The diagnosis of hiatus hernia is usually by barium meal examination. The incidence of hiatus hernia in any centre depends on the index of suspicion of the medical staff as well as on the efforts made by the radiologist to demonstrate it (Jones, 1952; Clagett, 1966). The low incidence of hiatus hernia reported from various parts of Africa could be a reflection of inadequate efforts of clinician and radiologist. In order to test this hypothesis we decided on a prospective study of the incidence of hiatus hernia and gastrooesophageal reflux in a series of consecutive barium meal examinations of patients attending the Lagos University Teaching Hospital. Al- though previous reports (Grech, 1965; Whittaker, 1966) had commented on the rarity of hiatus hernia in barium meal examination of Africans in Tanzania and Kenya respectively, they were based on retrospective studies and suffered from all the disadvantages of such studies, including possible lack of sufficient awareness of the condition. No previous prospective barium meal studies of the African have been undertaken with the sole purpose of establishing the incidence of hiatus hernia and gastro-oesophageal reflux.

\section{Material and method}

The patients were consecutive adult Nigerians referred for barium meal or barium swallow examinations by clinicians in the different outpatient clinics and clinical departments of the Lagos University Teaching Hospital in the period April 1970 to April 1972. All the patients had symptoms which, in the opinion of the referring slinician, warranted barium meal or barium swallow investigations. All patients were examined for the presence of hiatus hernia and gastrooesophageal reflux, irrespective of the clinical indications for their investigation, while at the same time the request of the referring clinician was complied with. 
The diagnosis of hiatus hernia was based on a combination of criteria adopted by Sommer and Stevenson (1961) and Sim (1964):

(a) demonstration of herniated loculus above the diaphragm in the general direction of the stomach below the diaphragm, in which gastric mucosa could be shown to be continuous with the gastric mucosa of the infradiaphragmatic portion of the stomach;

(b) demonstration of a distinct ring of demarcation at the upper border of the herniated loculus; and

(c) demonstration of reflux in a retrograde manner from the infradiaphragmatic stomach to fill the herniated loculus for at least $3 \mathrm{~cm}$ above the diaphragm.

At least two of the above criteria were to be present, and supportive evidence of hiatus hernia included the following:

(a) demonstration of obliterated gastro-oesophageal angle; and

(b) diminished or absent peristalsis in the sac which distinguished it from the region of active oesophageal peristalsis above.

As far as possible examinations were carried out during the morning. Patients were requested to starve from $21.00 \mathrm{~h}$ of the night preceding the examination. The $x$-ray assembly used for the study was a Watson Roentgen 600 generator and the Autonome 4 diagnostic table. Fluoroscopy was performed using an image intensifier coupled to a television screen.

After preliminary screening of the chest in the erect position, noting any loculus above the diaphragm containing air, the patient was examined standing in the left lateral position and given barium to drink. He was then screened while bending to touch his toes, and spot films were taken. The patient then stood up and the oesophagus was allowed to empty. He was then examined lying prone on the table with both hands firmly grasping its edges and his abdomen firmly applied to its surface. The table was mechanically tilted to the horizontal position and the patient was examined for the presence of a herniated loculus based on the defined criteria. Gastrooesophageal reflux was diagnosed if barium was noted in the previously empty oesophagus. While still in the prone position the patient swallowed more barium and was again screened for the presence of hiatus hernia and gastro-oesophageal reflux. If neither gastro-oesophageal reflux nor hiatus hernia was demonstrated the patient was requested to perform a Valsalva manoeuvre and the screening and spot film were repeated.
If these manoeuvres failed to demonstrate a hiatus hernia or gastro-oesophageal reflux the patient was screened in the right anterior oblique position with the trunk lifted slightly off the table. If these examinations were negative the patient was finally examined in a head-down prone position with the table tilted 10 to 15 degrees below the horizontal.

Some elderly patients who were unable to bend down were examined in the prone and head down positions only. Patients with congestive heart failure or severe respiratory problems, who were too ill to go through the vigorous procedure, had a straightforward barium swallow or meal and have therefore been excluded from the series.

During the screening and spot filming radiation of the patient was kept to a minimum, and the time taken for the study of the lower end of the oesophagus did not appreciably prolong the total time of the examination.

\section{Results}

A total of 1030 adult Nigerian patients were studied. There were 985 barium meals and 45 barium swallows. There were 648 men and 382 women with a male to female ratio of $1 \cdot 7: 1$. Clinical indications, obtained from the clinician's request forms, were known in 978 patients. In 52 patients the indication was not stated, although all patients, irrespective of whether or not the indication was known, went through the same routine. The majority of the patients with stated indications $(77.6 \%)$ were referred for barium meal examination because of clinical suspicion of peptic ulcer while $5.8 \%$ of the patients were referred because of suspicion of hiatus hernia. 'Other indications' included such conditions as 'noises in the throat', pharyngeal pouch, atrophic gastritis, and anaemia.

Out of the total of 1030 barium examinations there were 369 positive findings $(35.8) \%$ and 606 normal findings $(58.8 \%)$. In 55 patients $(5 \cdot 3 \%)$ the diagnosis was uncertain.

There were only four cases of hiatus hernia out of 1030 patients studied, representing $0.39 \%$ of all patients and $1.1 \%$ of all positive radiological findings.

There were 23 patients with gastro-oesophageal reflux representing $2 \cdot 2 \%$ of all patients examined. Only two out of 23 patients with gastro-oesophageal reflux had a sliding hiatus hernia. Eleven patients (eight women, three men) had reflux without any associated condition. The average age of the 11 patients who had reflux but no demon- 
strable hernia was $40 \cdot 7$ years, 10 years less than that of the four patients with hiatus hernia in whom the average age was 51 years. One patient had an oesophageal diverticulum and another patient had a hepatoma.

The remaining eight patients among those who had gastro-oesophageal reflux also had peptic ulcers and appear to differ from the patients described above. There were seven males and one female, a male preponderance similar to that shown by all the patients with peptic ulcer. Out of the 259 patients with peptic ulcer there were 201 males and 58 females, a male to female ratio of $3.4: 1$. It would thus appear that the gastrooesophageal reflux found in association with peptic ulcer has a different aetiology from gastro-oesophageal reflux related to other conditions.

\section{Discussion}

Although the authors cited above had commented on the low incidence of hiatus hernia in Africans no authoritative study existed to confirm this clinical impression. This prospective study has confirmed that the incidence of both hiatus hernia and gastro-oesophageal reflux is very low in Nigerians. Only four cases of hiatus hernia, an incidence of $0.39 \%$, were found in a study deliberately undertaken to determine the incidence of hiatus hernia and gastro-oesophageal reflux. These were symptomatic patients, and an incidence of $0.39 \%$ in a study of this nature is very low when compared with studies of symptomatic patients in Europe and North America. There, the incidence of hiatus hernia in barium meal studies in both symptomatic and asymptomatic people varies between $30 \%$ and $56 \%$ (Stein and Finkelstein, 1960; Vandervelde and Carlson, 1964; Pridie, 1966; Dyer and Pridie, 1968; Mandelstam and Lieber, 1970).

The incidence of gastro-oesophageal reflux in our series was equally low. There were only 23 patients $(2 \cdot 2 \%)$ with gastro-oesophageal reflux, only two of whom had sliding hiatus hernia. Gastro-oesophageal reflux found in the eight patients who also had duodenal ulcers appears to have a different aetiology. The reflux in these cases seems to depend on gastric outlet obstruction, referred to by Burge et al. (1966) as pyloric channel disease. The incidence of gastro-oesophageal reflux in Caucasian duodenal ulcer patients varies between $28 \%$ and $50 \%$ (Casten et al., 1963; Clarke et al., 1965; Burge et al., 1966). Burge et al. (1966) cured the reflux in this group of patients by an operation to relieve the outlet obstruction. Pyloric stenosis is the commonest complication of duodenal ulcer and the second commonest indication for operation in duodenal ulcer patients in Nigeria (Davey, 1968; Ogunbiyi and Akinhanmi, 1973). It seems reasonable to expect that many of the Nigerian duodenal ulcer patients will have various grades of gastric outlet obstruction, and yet the incidence of gastrooesophageal reflux associated with duodenal ulcer was only $3.1 \%$ in our study, as against $28 \%$ and $50 \%$ in Caucasians.

The low incidence of hiatus hernia and gastrooesophageal reflux is a feature of barium meal studies carried out in Africans by other workers. Grech (1965) found only two cases of hiatus hernia, one of which was para-oesophageal while the other was traumatic, in a series of 733 barium meal examinations of Africans in Tanzania. Whittaker (1966) found only one case of hiatus hernia in 193 barium swallows and 1319 barium meals in Africans in Kenya. One of us, in a separate series, found only two cases of hiatus hernia and 34 cases of gastro-oesophageal reflux in barium meal examinations of 657 symptomatic Nigerians (Eyo, 1973).

Edwards (1971) has remarked that if barium can be seen to reflux consistently when the subject lies down, turns to one side or bends to touch his toes, then the anti-reflux mechanism is faulty in the circumstances of the examination. Failure to demonstrate reflux, even in circumstances in which it occurs very commonly in other races, must indicate that there is some characteristic of the gastro-oesophageal mechanism in Nigerians that makes their lower oesophageal sphincter, the most important anti-reflux mechanism, particularly competent.

We acknowledge with gratitude the assistance of Mr. J. A. Somoye, chief radiographer, and of Mrs. 'Shade Olatunji in assembling the radiographic reports.

Professor E. A. Elebute, Head of the Department of Surgery, assembled the data during the closing period of this study when one of the authors (OOB) was away. We remain very grateful to him and to Dr. F. E. U. Isangedighi who assisted him.

This communication forms part of a work submitted to and accepted by the University of London for the degree of Master of Surgery.

\section{References}

Atkinson, M. (1967). Hiatus hernia. British Medical Journal, 4, 218-221. 
Barrett, N. R. (1952). Discussion on hiatus hernia. Proceedings of the Royal Society of Medicine, 45, 279-286.

Bassey, O. O. (1975). Manometric study of the lower oesophageal sphincter in adult Nigerians as a possible explanation for the observed low incidence of hiatal hernia and symptomatic gastro-oesophageal reflux. MS thesis, London University, May 1975.

Beeley, M. and Warner, J. O. (1972). Medical treatment of symptomatic hiatus hernia with low-density compounds. Current Medical Research and Opinion, 1, 63-69.

Burge, H. W., Gill, A. M., Maclean, C. D. T., and Lewis, R. H. (1966). Symptomatic hiatus hernia. Thorax, 21, 67-74.

Burkitt, D. P. (1971). Personal communication.

Burkitt, D. P. and James, P. A. (1973). Low-residue diets and hiatus hernia. Lancet, 2, 128-130.

Casten, D. F., Bernhang, A., Nach, R. J., and Spinzia, J. (1963). A physiological basis for the surgical treatment of sliding esophageal hiatal hernia. Surgery, Gynecology and Obstetrics, 117, 87-93.

Clagett, O. T. (1966). Present concepts regarding the surgical treatment of oesophageal hiatal hernia. Annals of the Royal College of Surgeons of England, 38, 195-209.

Clarke, S. D., Penry, J. B., and Ward, P. (1965). Oesophageal reflux after abdominal vagotomy. Lancet, 2, 824-826.

Davey, W. W. (1968). Companion to Surgery in A frica, p. 185. Livingstone, Edinburgh and London.

Dyer, N. H. and Pridie, R. B. (1968). Incidence of hiatus hernia in asymptomatic subjects. Gut, 9, 696-699.

Edwards, D. A. W. (1971). The oesophagus. Gut, 12, 948-956.

Eyo, E. E. (1973). Personal communication.

Grech, P. (1965). Radiological analysis of lesions of the upper intestinal tract during a four-year period in Africans in Tanganyika (now Tanzania). East A frican Medical Journal, 42, 106-116.

Jones, F. A. (1952). Discussion on hiatus hernia: diagnosis of hiatus hernia. Proceedings of the Royal Society of Medicine, 45, 277-279.

Mandelstam, P. and Lieber, A. (1970). Cineradiographic evaluation of the esophagus in normal adults: a study of 146 subjects ranging in age from 21 to 90 years. Gastroenterology, 58, 32-39.

Ogunbiyi, T. A. J. and Akinhanmi, G. A. (1973). Pyloric stenosis in adults. Nigerian Medical Journal, 3, 24-31.

Pridie, R. B. (1966). Incidence and coincidence of hiatus hernia. Gut, 7, 188-189.

Sim, G. P. A. (1964). An evaluation of tests for hiatus hernia. British Journal of Radiology, 37, 781-787.

Sommer, A. W. and Stevenson, C. L. (1961). Hiatal hernia: an evaluation of diagnostic procedures. American Journal of Digestive Diseases, 6, 412-422.

Stein, G. N. and Finkelstein, A. (1960). Hiatal hernia: roentgen incidence and diagnosis. American Journal of Digestive Diseases, 5, 77-87.

Trowell, H. C. (1960). Non-infective Disease in Africa, p. 197. Edward Arnold, London.

Vandervelde, G. M. and Carlson, H. C. (1964). Esophageal reflux. American Journal of Roentgenology, Radium Therapy and Nuclear Medicine, 92, 989-993.

Whittaker, L. R. (1966). A review of a series of radiological examinations of the upper alimentary tract in African patients. East African Medical Journal, 43, 336-340.

Requests for reprints to: O. O. Bassey, MS, FRCS, Department of Surgery, College of Medicine, University of Lagos, PMB, 12003, Lagos, Nigeria. 